$04 ; 12$

\title{
Вариации параметров пучка убегающих электронов в газовом разряде в условиях неоднородной предварительной \\ ионизации
}

\author{
(C) А.В. Козырев ${ }^{1,2}$, Е.М. Баранова ${ }^{1}$, В.Ю. Кожевников ${ }^{1,2}$, \\ Н.C. Семенюк ${ }^{1,2}$ \\ ${ }^{1}$ Томский государственный университет \\ ${ }^{2}$ Институт сильноточной электроники СО РАН, Томск \\ E-mail: kozyrev@to.hcei.tsc.ru
}

Поступило в Редакцию 3 мая 2017 г.

В рамках гибридной теоретической модели наносекундного разряда высокого давления с убегающими электронами продемонстрирована сильная зависимость амплитуды, длительности и энергетического спектра электронного пучка от условий предварительной ионизации газа в промежутке. Смоделированы ситуации с однородным и резко неоднородными распределениями инициирующих электронов в коаксиальном диоде, заполненном гексафторидом серы атмосферного давления. Показано, что амплитуда и профиль импульса тока электронного пучка существенно изменяются при вариации начального распределения электронов в промежутке.

DOI: $10.21883 /$ PJTF.2017.17.44947.16848

В последние годы низкотемпературная плазма, генерируемая в газовом разряде атмосферного давления, все чаще используется в различных технических приложениях. Генерация убегающих электронов в газовом разряде высокого давления является предметом большого числа экспериментальных и теоретических работ, ссылки на которые можно найти в научных обзорах и монографиях [1-5], посвященных этому явлению. Физическая природа появления быстрых электронов в газовом разряде состоит в том, что при высоких отношениях напряженности электрического поля к давлению часть электронов может переходить в режим непрерывного ускорения. Высокие приведенные напряженности на уровне $E / P>200 \mathrm{~V} /(\mathrm{cm}$. Torr) имеют место в течение короткого 
момента времени на начальной стадии пробоя газа, когда на газоразрядный промежуток с малым радиусом кривизны катода подается импульс высокого напряжения с субнаносекундной длительностью переднего фронта. Ярко выраженная нестационарность и неоднородность разряда в таких условиях делают как теоретические, так и экспериментальные исследования весьма затруднительными. Широкое разнообразие начальных условий пробоя, конструкций разрядной камеры и методов регистрации коротких импульсов тока быстрых электронов приводит, как правило, к большому разбросу измеряемых параметров у разных исследовательских групп [1-4]. Мы полагаем, что большой разброс измеряемых параметров импульсов тока и спектра быстрых электронов в экспериментах подчас обусловлен таким трудноконтролируемым фактором, как начальное распределение электронов, имеющее место в промежутке до прихода импульса напряжения. Этот фактор имеет большую флуктуацию даже при полностью контролируемых остальных условиях эксперимента. В настоящей работе на основе оригинальной теоретической модели мы демонстрируем влияние начальных режимов предварительной ионизации газа на параметры наносекундного разряда и импульса тока убегающих электронов.

Ранее авторами была разработана и успешно апробирована гибридная модель описания разряда с убегающими электронами, которая впервые позволила количественно рассчитать наблюдаемые характеристики пучка быстрых электронов, генерируемого при пробое гексафторида серы высокого давления [6,7]. Два совершенно разных метода математического описания различных подсистем разряда составляют суть гибридной модели. Полагая, что доля электронов с высокой энергией всегда незначительна и почти не влияет на динамику разряда, весь расчет можно разделить на две последовательные задачи: описание самого разряда и формирование потока убегающих электронов. Первая задача позволяет описывать поведение электронионных компонент низкотемпературной плазмы разряда в дрейфоводиффузионном приближении. Затем с использованием известных в каждый момент времени распределений напряженности электрического поля и источника ионизации газа решается кинетическое уравнение Больцмана для функции распределения убегающих электронов по скоростям (импульсам).

В качестве упрощенной модели разряда с неоднородной геометрией разрядного промежутка, допускающей математическое описание на

Письма в ЖТФ, 2017, том 43, вып. 17 


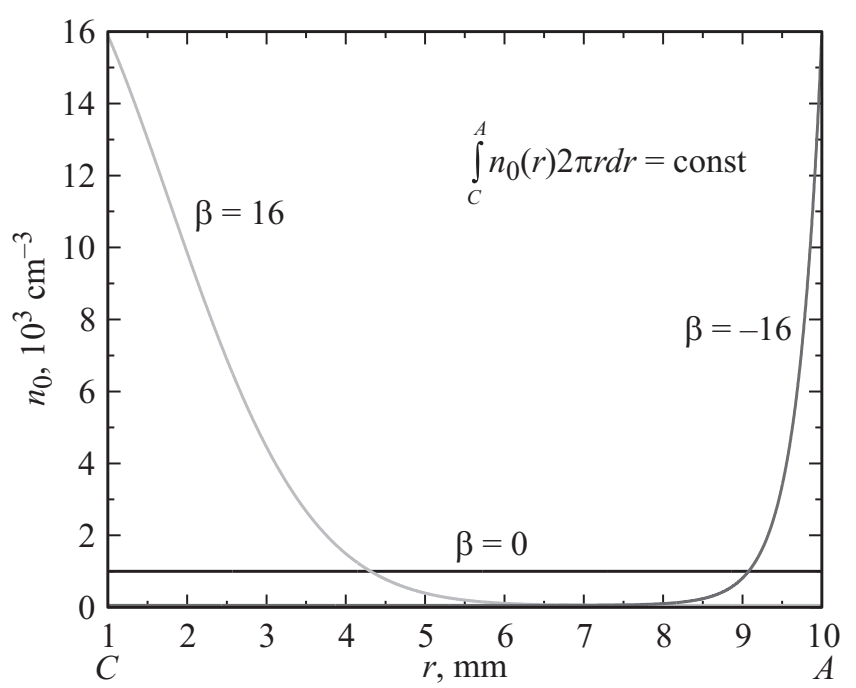

Рис. 1. Пространственное распределение начальной концентрации электронов в коаксиальном промежутке при различных значениях параметра $\beta$.

основе уравнений в частных производных, был выбран коаксиальный газонаполненный диод (давление газа $P$, радиус катода $r_{C}$, радиус анодного цилиндра $r_{A}$, продольная длина разрядной трубки $L$ ). На такой промежуток, включенный в последовательную электрическую цепь с источником напряжения и балластным сопротивлением $R$, подается электрический импульс $U_{S}(t)$ с крутым передним фронтом и амплитудой, существенно превышающей напряжение статического пробоя газа.

Начальная концентрация электронов $n_{0}$ задавалась на низком уровне так, чтобы радиальная зависимость начальной концентрации описывалась гауссовой функцией $n_{0}(r) \propto \exp \left(-\beta r^{2} / r_{A}^{2}\right)$, а полное число инициирующих электронов в межэлектродном пространстве не менялось при вариации параметра $\beta$. Радиальные зависимости $n_{0}(r)$ при трех значениях параметра $\beta$ показаны на рис. 1, радиус катода равен $r_{C}=1 \mathrm{~mm}$, радиус анода $r_{A}=10 \mathrm{~mm}$. При величинах $\beta= \pm 16$ начальная концентрация у одного из электродов на семь порядков отличалась от концентрации у второго! Поэтому при больших положительных $\beta$

Письма в ЖТФ, 2017, том 43, вып. 17 
можно говорить о прикатодной, а при больших отрицательных $\beta-$ о прианодной предварительной ионизации, при $\beta=0$ начальная предварительная ионизации была однородной.

Для описания пространственно-временно́й эволюции плотности плазменных электронов $n_{e}(r, t)$ и напряженности электрического поля $E(r, t)$ в промежутке используются три уравнения: уравнение непрерывности для электронной компоненты плазмы

$$
\frac{\partial n_{e}}{\partial t}=-\frac{1}{r} \frac{\partial}{\partial r}\left\{r\left(w_{e} n_{e}-D_{e} \frac{\partial n_{e}}{\partial r}\right)\right\}+(\alpha-\eta) w_{e} n_{e}
$$

уравнение сохранения для погонной плотности радиального тока в электрической цепи $J(t)$

$$
\varepsilon_{0} \frac{\partial E}{\partial t}=\frac{J(t)}{2 \pi r}+e\left(w_{e} n_{e}-D_{e} \frac{\partial n_{e}}{\partial r}\right)
$$

уравнение Кирхгофа для электрической цепи

$$
J(t)=\frac{1}{L R}\left(U_{0}(t)-\int_{r_{C}}^{r_{A}} E(r, t) d r\right) .
$$

Здесь $\alpha(E), \eta(E), w_{e}(E)$ - коэффициент ионизации Таунсенда, коэффициент прилипания и скорость дрейфа плазменных электронов соответственно как известные функции локальной напряженности поля, $D_{e}-$ коэффициент электронной диффузии, $e-$ элементарный заряд, $\varepsilon_{0}$ - электрическая постоянная. В результате решения системы $(1)-(3)$ мы получали три функции: $n_{e}(r, t), E(r, t)$ и $J(t)$, которые полностью характеризовали „анатомию“ разряда. Интегрированием $E(r, t)$ по координате рассчитывалось мгновенное падение напряжения на диоде $U(t)$.

Формирование неоднородной функции распределения убегающих электронов по импульсам $f(r, p, t)$ описывалось релятивистским уравнением Больцмана с модельной правой частью

$$
\gamma\left(\frac{\partial f}{\partial t}+\frac{p}{m \gamma} \frac{\partial f}{\partial r}-e E(r, t) \frac{\partial f}{\partial p}\right)=\alpha w_{e} n_{e} f_{0}(p)-n_{g} \frac{p}{\tilde{m} \gamma} \sigma^{*}(p) f(p) .
$$

Здесь $m-$ масса покоя электрона, $p-$ релятивистский импульс, $\gamma=\sqrt{1+(p / m c)^{2}}-$ релятивистский фактор, $c-$ скорость света,

Письма в ЖТФ, 2017, том 43, вып. 17 


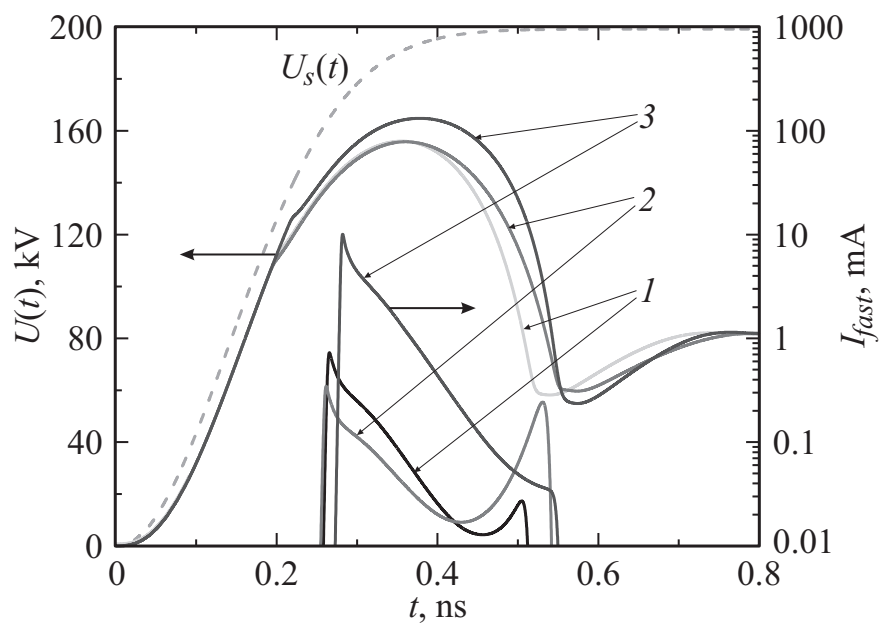

Pис. 2. Зависимость от времени напряжения источника питания $U_{s}(t)$, напряжения на диоде и тока быстрых электронов за анодом при разных распределениях инициирующих электронов: $1-\beta=0$ (однородная предварительная ионизация), $2-\beta=16$ (прикатодная предварительная ионизация), $3-\beta=-16$ (прианодная предварительная ионизация).

$\sigma^{*}(p)$ - транспортное сечение столкновений электрона с атомами как функция импульса, $n_{g}-$ концентрация атомов газа, $f_{0}(p)-$ максвелловская функция распределения плазменных электронов. Первое слагаемое в правой части описывает появление плазменных электронов в промежутке за счет ударной ионизации газа, а второе - „гибель“ убегающих электронов в рассеивающих столкновениях. Главным достоинством нашей модели является то, что в уравнение (4) мы подставляем уже вычисленные из (1)-(3) функции $n_{e}(r, t)$ и $E(r, t)$.

На рис. 2 приведены вычисленные зависимости от времени напряжения горения разряда и тока быстрых электронов для следующей конкретной задачи. В коаксиальном диоде (радиус катода $r_{C}=1 \mathrm{~mm}$, радиус анода $r_{A}=10 \mathrm{~mm}$, погонная длина $L=1 \mathrm{~cm}$ ) развивается разряд в $\mathrm{SF}_{6}$ при давлении $10^{5} \mathrm{~Pa}$. Импульс напряжения амплитудой $200 \mathrm{kV}$ с длительностью переднего фронта $0.3 \mathrm{~ns}$ по уровню $0.1-0.9$ от максимального поступал на диод, последовательно с которым включено 


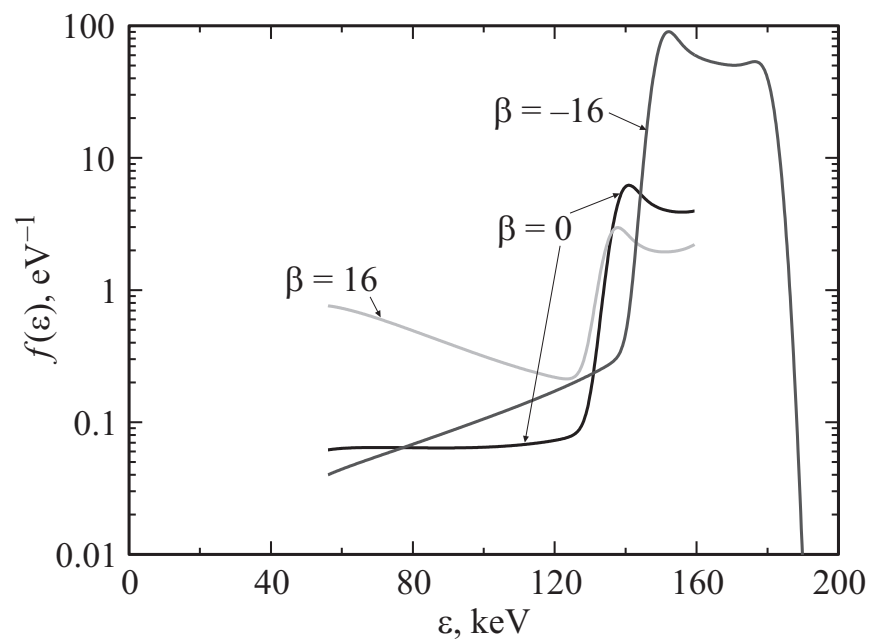

Рис. 3. Интегральные (за один импульс) энергетические спектры быстрых электронов за анодной фольгой.

балластное сопротивление $R=75 \Omega$. Параметры элементарных процессов $\left(\alpha, \eta, w_{e}, D_{e}\right)$ в системе (1)-(3) для $\mathrm{SF}_{6}$ взяты из работы [8], а транспортные сечения $\sigma^{*}$ для уравнения (4) - из работы [9]. Для того чтобы расчеты убегающих электронов можно было сравнивать с экспериментальными данными, мы использовали энергетический фильтр в виде алюминиевой фольги толщиной $d=10 \mu \mathrm{m}$, отсекающий приходящие на анод из столба разряда медленные электроны.

Как видно из рис. 2, количество быстрых электронов в случае „прианодного“ инициирования примерно на порядок выше, чем при других режимах подсветки (обратим внимание на логарифмический масштаб шкалы для токов пучка). Это напрямую связано с большей амплитудой напряжения на промежутке, а следовательно, и большей вероятностью перехода плазменных электронов в режим непрерывного ускорения. Кроме того, в режиме прикатодного инициирования ток пучка состоит из двух импульсов: первый связан приходом на анод опережающей группы убегающих электронов, рожденных на ранней стадии пробоя в области плотной прикатодной плазмы, а второй

Письма в ЖТФ, 2017, том 43, вып. 17 
импульс обусловлен приходом на анод фронта плотной плазмы разряда. Средняя энергия электронов во втором импульсе тока существенно ниже, чем в первой группе. Это демонстрируется на рис. 3, где показаны энергетические спектры быстрых электронов для трех рассматриваемых режимов подсветки. Как видно, в режиме „прикатодного“ инициирования спектр быстрых электронов довольно широкий и в отличие от двух других режимов подсветки имеет двухмодовую структуру. Наши расчеты на качественном уровне хорошо согласуются с результатами экспериментов из работы [10], где в субнаносекундном разряде в диоде со стержневым катодом отчетливо наблюдалась двухмодовая форма импульса тока и энергетического спектра убегающих электронов.

Таким образом, на основе гибридной физико-математической модели газового разряда с убегающими электронами выявлено сильное влияние режима предварительной ионизации на параметры пучка убегающих электронов. В частности, „прикатодное“ инициирование разряда может приводить к двухмодовой структуре импульса тока пучка. В лабораторных приборах неоднородность предварительной ионизации, вызванная флуктуациями параметров источника подсветки, временем рекомбинации остаточной плазмы или особенностями конструкции диода, может меняться в широких пределах. Нам кажется, что именно по этой причине наблюдаемые в экспериментах флуктуации параметров пучка быстрых электронов очень велики даже в одной и той же серии экспериментов.

Работа выполнена при поддержке РФФИ (проект № 17-08-00932-а).

\section{Список литературы}

[1] Бабич Л.П., Лойко Т.В., Цукерман В.А. // УФН. 1990. Т. 160. № 7. С. 49-82.

[2] Runaway electrons preionized diffuse discharges / Ed. V.F. Tarasenko. N.Y.: Nova Science Publ., Inc., 2014. 598 p.

[3] Месяи, Г.А., Шпак В.Г., Шунайлов С.А., Яландин М.И. // Письма в ЖТФ. 2008. T. 34. B. 4. C. $71-80$.

[4] Levko D., Krasik Ya.E. // J. Appl. Phys. 2012. V. 111. Iss. 1. P. 013305.

[5] Лисенков В.В., Шкляев В.А. // Письма в ЖТФ. 2013. Т. 39. В. 16. С. 38-46.

[6] Kozyrev A.V., Kozhevnikov V.Yu., Lomaev M.I., Sorokin D.A., Semeniuk N.S., Tarasenko V.F. // Europhys. Lett. 2016. V. 114. N 4. P. 45001.

[7] Kozyrev A.V., Kozhevnikov V.Yu., Semeniuk N.S. // Matter Rad. Extremes. 2016. V. 1. N 5. P. 264-268.

Письма в ЖТФ, 2017, том 43, вып. 17 
[8] Dhali S.K., Pal A.K. // J. Appl. Phys. 1988. V. 63. Iss. 5. P. 1355-1362.

[9] Christophorou L.G., Olthon J.K. // J. Phys. Chem. Ref. Data. 2000. V. 29. N 3. P. 267-330.

[10] Gurevich A.V., Mesyats G.A., Zybin K.P., Yalandin M.I., Reutova A.G., Shpak V.G., Shunailov S.A. // Phys. Rev. Lett. 2012. Iss. 8. V. 109. P. 085002.

Письма в ЖТФ, 2017, том 43, вып. 17 